\title{
An organometallic ruthenium nanocluster with conjugated aromatic ligand skeleton for explosive sensing
}

\author{
PRANAV DAVE, BHAVESH AGRAWAL, JAYDEV THAKARDA, SAGAR BHOWMIK and \\ PRASENJIT MAITY*iD \\ Institute of Research and Development, Gujarat Forensic Sciences University, Gandhinagar, Gujarat 382007 , \\ India \\ E-mail: pmaity@gfsu.edu.in
}

MS received 14 November 2018; revised 14 December 2018; accepted 21 December 2018; published online 30 January 2019

\begin{abstract}
Ethynylphenanthrene (EPT) bound to highly monodispersed Ruthenium (Ru) nanocluster (Ru:EPT) with mean diameter of $1.5 \pm 0.2 \mathrm{~nm}$ and mol wt. of $\sim 8600 \mathrm{Da}$ was synthesized via a facile and high yield biphasic ligand exchange protocol using similar sized ethylene glycol (EG)-stabilized Ru clusters (Ru:EG) as precursor. The synthesized organometallic nanocluster was meticulously analyzed to understand its size distribution, oxidation state, crystallinity, optical and luminescence behavior and metal-ligand interfacial structure. Contrary to the extensive quenching of ligand emission by metalcore as usually observed, the ruthenium core here acts as a conductor, which conjugates surface ligands with strong emission property courtesy to an unusual vinylidene-binding motif. Thus, the synthesized nanocluster shows good luminescence property $(\phi=\sim 7 \%)$ originated from the ligand skeleton and the spherical metal core restricts lateral overlap of phenanthrene moiety to cause any excimer emission. This nanocluster showed high sensitivity for solution phase detection of nitroaromatic explosives through luminescence quenching method $\left(\mathrm{K}_{\mathrm{SV}}\right.$ up to $\left.4.98 \times 10^{4} \mathrm{M}^{-1}\right)$ and mimic the mechanism like conjugated organic polymer. We propose that dynamic $\pi-\pi$ interaction between Ru bound phenanthrene moiety and nitroaromatic compounds followed by photoinduced electron transfer (PET), as well as Förster Resonance Energy Transfer (FRET), are the possible mechanisms behind this luminescence quenching.
\end{abstract}

Keywords. Nanocluster; Organometallic; Ruthenium; Explosive sensing; Photoluminescence.

\section{Introduction}

Organic ligand-stabilized metal clusters and nanoparticles are an important class of materials due to their size-specific physicochemical properties including unique optical, emission, catalytic, magnetic and electronic properties. ${ }^{1-4}$ The use of various organic ligands including phosphine, amine, carboxylate, thiol and selenol has been explored extensively for the stabilization of metal clusters. In recent years carbon-based ligands (e.g., alkyne, alkene, carbene, etc.) have been successfully used to synthesize different metal clusters and nanoparticles. ${ }^{5-13}$ Contrary to other ligands as mentioned above, carbon-based ligands are attractive candidates because we could expect better electronic conjugation between ligand skeleton and metal core through conjugated metal-carbon bond. This anticipated electronic conjugation will substantially alter the electronic nature of the metal cluster and surface ligands and could prove valuable for catalysis ${ }^{14}$ and nanoelectronics applications. ${ }^{15}$ The subsequent effect on the luminescence behavior of the cluster is also an interesting phenomenon to be studied and has the potential for application in sensing different molecules specific to attached ligand-based receptor. However, the synthesis of such luminescent metal clusters with controlled chemical scaffolds and their application in sensing is a challenging task. It is challenging because metal clusters with luminescent ligand results strong charge transfer from ligand to metal and thus quenching of emission is inevitable. ${ }^{16}$ However, if a properly oriented ligand with intense luminescence property could be held firmly on

\footnotetext{
*For correspondence 
the curved metal surface, then it would be interesting for application in luminescence-based sensing.

Polynitroaromatic molecules e.g., Dinitrotoluene (DNT), Trinitrotoluene (TNT), Trinitrophenol (TNP) are extensively used as secondary explosive materials. The development of reliable, cost-effective and simple technology for the detection of these nitroaromatic explosives is very essential. Luminescence quenching based technique ${ }^{17-28}$ for explosive detection is very attractive due to its high sensitivity, easy portability, quick response and inexpensive processing cost. Conjugated organic/inorganic polymers ${ }^{17,18}$ and $\pi$-electron rich small molecules ${ }^{24}$ have been used extensively for trace level detection of explosives. Ruthenium metal-based cluster and metal-organic frameworks have also been demonstrated for the detection of explosive molecules. ${ }^{27,28}$ In case of the conjugated polymer, a single explosive molecule can quench the luminescence of whole polymer chain, thus they are preferred for better sensitivity over a small molecule (e.g., pyrene) where, the only a fraction of molecules interacting with explosives is quenched, whereas rest of the molecules remain luminescent. On the contrary, small molecules are preferred over polymers for solution phase explosive sensing study due to better control of their solubility by modifying surface scaffolds. The replication of the sensing behavior of conjugated polymer system in a ligand-stabilized metal cluster will be interesting and appealing for explosive sensing viewpoint. The syntheses routes of various organometallic clusters and nanoparticles have been developed by Tsukuda and Maity, ${ }^{8-12}$ Schriffrin, ${ }^{5}$ Chen, ${ }^{6,7}$ and Wang ${ }^{13}$ in recent years via self-assembly, base-promoted direct ligation and ligand exchange based strategies with a major focus on gold clusters. In the present work, we are reporting the synthesis of an alkyne based pi electronrich aromatic ligand (9-ethynylphenanthrene) stabilized $\mathrm{Ru}$ cluster via facile ligand exchange strategy and its detail characterization including molecular weight determination through MALDI mass spectrometry. The $\mathrm{Ru}$ nanocluster showed good luminescence behaviour through intra-ligand electronic conjugation and showed high sensitivity for detection of electron-deficient explosive molecules through solution phase luminescence based titration method.

\section{Experimental}

\subsection{Materials and methods}

All the reagents were commercially available and were used without further purification. Ruthenium trichloride
$\left(\mathrm{RuCl}_{3}\right)$, 9-ethynylphenanthrene (EPT), trans-2-[3-(4-tertButylphenyl)-2-methyl-2propenylidene] malonitrile (DCTB), Sephadex LH-20 resin, sodium tetrahydroborate, sodium hydroxide, methanol, toluene, chloroform and ethylene glycol were obtained from Sigma Aldrich. Milli-Q grade water was used in the present study. UV-visible spectra of respective clusters were obtained in water or chloroform as solvent using a JASCO V-670 spectrophotometer. Photoluminescence spectra were obtained in chloroform using a JASCO FP6600 spectrofluorometer. TEM images were recorded using a Philips JEM 2000FX microscope operated at $200 \mathrm{kV}$. FT-IR spectra were obtained using a JASCO FT/IR-4200 spectrophotometer with samples prepared as $\mathrm{KBr}$ pellets. XPS measurements were performed using a JEOL, JPS9010MC spectrometer with an $\mathrm{Mg} \mathrm{K} \alpha$ source. The spectrum was calibrated relative to the $\mathrm{C}_{1 s}$ peak, which was adjusted to the energy of $284.5 \mathrm{eV}$. PXRD pattern was measured using a GNR-APD 2000 advance diffractometer with a $\mathrm{Cu}$ $\mathrm{K} \alpha$ source. Emission quantum yields were measured by the method of Demas and Crosby ${ }^{29}$ with $\left[\mathrm{Ru}(\mathrm{bpy})_{3}\right]\left(\mathrm{PF}_{6}\right)_{2}$ as the standard $\left(\Phi_{\text {ref }}=0.062\right)$. MALDI mass spectrum was recorded by using an Applied Biosystems (4800 plus MALDI TOF) instrument using $\mathrm{N}_{2}$ diode laser. Photoluminescence (PL) lifetime measurement was carried out by a picosecond time-correlated single photon counting (TCSPC) instrument (Edinburgh Instruments Ltd, Lifespec II model).

[Some explosive samples (TNT, RDX and PETN) were provided by Directorate of Forensic Science Laboratory, Gandhinagar, India and were used in small quantities with extreme care.]

\subsection{Synthesis of ethylene glycol (EG) stabilized Ru Clusters}

The synthesis was followed by procedure reported by Wang et al. ${ }^{30}$ In brief, Ruthenium trichloride $\left(\mathrm{RuCl}_{3}\right)(0.1 \mathrm{mmol})$ was dissolved in $15 \mathrm{~mL}$ ethylene glycol along with $0.5 \mathrm{mmol}$ of sodium hydroxide. The solution mixture fitted with a magnetic stirrer and heated at $160^{\circ} \mathrm{C}$ for $2 \mathrm{~h}$ with a constant flow of Argon on the open top of the flask to remove the water vapours. During this time, the solution colour changed to deep brown, indicating the formation of the nanoparticle. The solution was cooled, characterized by TEM and UV-Vis study and used as precursor clusters for further synthesis in the next step.

\subsection{Synthesis of EPT ligated Ru Cluster}

The EG stabilized Ru cluster synthesized in the previous step was taken and mixed with $5 \mathrm{mmol}$ of EPT in $15 \mathrm{~mL}$ chloroform. Then the solution was kept under stirring at $333 \mathrm{~K}$ for $3 \mathrm{~h}$. Due to ligand exchange, the cluster transfers from aqueous (EG) to the organic phase, which was then purified by solvent removal, followed by repeated washing with methanol and centrifugal precipitation. The purified and dried alkyne protected Ru cluster appears like deep brown to black, 


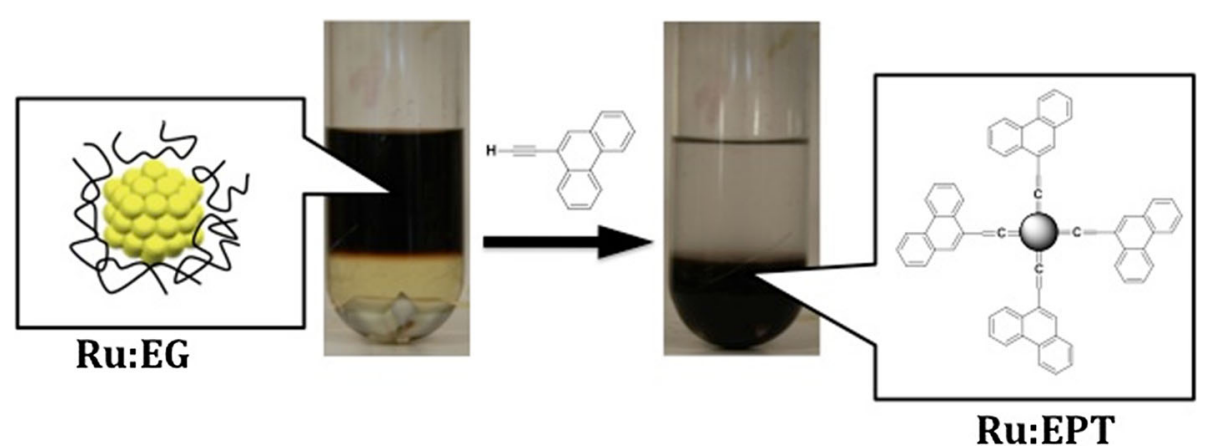

Scheme 1. Synthesis of Ru:EPT from Ru:EG by ligand exchange reaction and with the vinylidene binding motif of alkyne to $\mathrm{Ru}$.

soluble in apolar organic solvents (chloroform, toluene), but insoluble in polar solvents $(\mathrm{MeOH}$, water, acetone). Finally, the cluster was purified by size exclusion chromatography using Sephadex LH-20 as stationary phase and chloroform as mobile phase.

\subsection{Fluorescence sensing of explosive molecules}

Fluorescence titration was performed by mixing nanocluster and nitroaromatic compound in successive proportions in dichloromethane (DCM) followed by measuring their emission spectra. In brief, sequential addition of $10^{-4} \mathrm{M}$ each of explosives $(10 \mu \mathrm{L}$ in each time) to the solution of nanocluster (0.2 $\mathrm{mg}$ in $3 \mathrm{~mL}$ DCM) was carried out at room temperature. The fluorescence spectra were then measured and peak area was calculated for respective samples.

\section{Results and Discussion}

\subsection{Synthesis and purification of Ru:EPT cluster}

The synthesis of organometallic Ru:EPT cluster was performed by following the biphasic ligand exchange protocol (Scheme 1). The synthetic mechanism involves catalytic activation of acetylenic $\mathrm{C}-\mathrm{H}$ bond by $\mathrm{Ru}$ clusters followed by the formation of metal-carbon interfacial structure. The progress of the reaction was monitored through phase separation of the cluster from water to chloroform phase. The UV-Vis spectra of chloroform phase showed a continuous rise of absorption value with time in near-UV region confirming the phase separation and formation of lipophilic Ru:EPT cluster (Figure 1a). Finally, the cluster was purified by phase separation followed by size exclusion column chromatography to isolate the major monodispersed fraction of the cluster. The isolated and purified cluster was characterized by different spectroscopic and microscopic techniques (TEM, UV-Vis, PL, FT-IR, XRD, XPS and
MALDI) to understand its true identity and application potential.

\subsection{Size, interfacial structure and chemical state of Ru cluster}

The particle size and size distribution of the clusters were evaluated by comparing their respective TEM images and bar chart diagrams (Figures 1c-d). The average size of $1.4 \pm 0.2 \mathrm{~nm}$ of parent Ru:EG clusters gets converted to $1.5 \pm 0.2 \mathrm{~nm}$ sized organometallic cluster; which implies that the synthesis strategy preserves the size distribution greatly. The vibrational spectra (FTIR) of free ligand (EPT) and corresponding Ru cluster $(\mathrm{Ru}: \mathrm{EPT})$ is shown in Figure 1b. The characteristic peak of acetylenic hydrogen $(\equiv \mathrm{C}-\mathrm{H})$ at $3300 \mathrm{~cm}^{-1}$ is missing in the spectrum of the cluster, implying successful activation of this bond and its attachment to $\mathrm{Ru}$ surface. The drastic redshift of $-\mathrm{C} \equiv \mathrm{C}-$ of over $250 \mathrm{~cm}^{-1}$ in the cluster as compared to free ligand suggests that substantial electron shift from 'sp' hybridized acetylenic carbon to metal has taken place. Also significant is the presence of vibrational peaks at $3000 \mathrm{~cm}^{-1}$, which is characteristic peaks for $\mathrm{C}\left(\mathrm{sp}^{2}\right)-\mathrm{H}$ bonds.

The ${ }^{1} \mathrm{H}$ NMR spectrum of EPT and Ru:EPT were also measured (Figure 2a) to understand the metal-ligand interfacial structure. The acetylenic $\mathrm{C}-\mathrm{H}$ peak of ligand at $3 \mathrm{ppm}$ completely disappears in the cluster implying the breaking of this bond and its attachment to $\mathrm{Ru}$ surface. The strong aromatic $\mathrm{C}-\mathrm{H}$ peaks of EPT in the $7-8.5 \mathrm{ppm}$ region is significantly broadened, diminished and markedly down-shifted to $\sim 7.00 \mathrm{ppm}$. This is due to slowed tumbling of the nanoparticles and attachment of ligand on diverse binding sites on the metal surface. ${ }^{31}$ The oxidation state of ' $\mathrm{Ru}$ ' in the cluster was examined by measuring XPS spectrum of the cluster as a disk and then peak positions were calibrated with respect to $C_{1 s}$ peak at $284.5 \mathrm{eV}$. We could observe the 

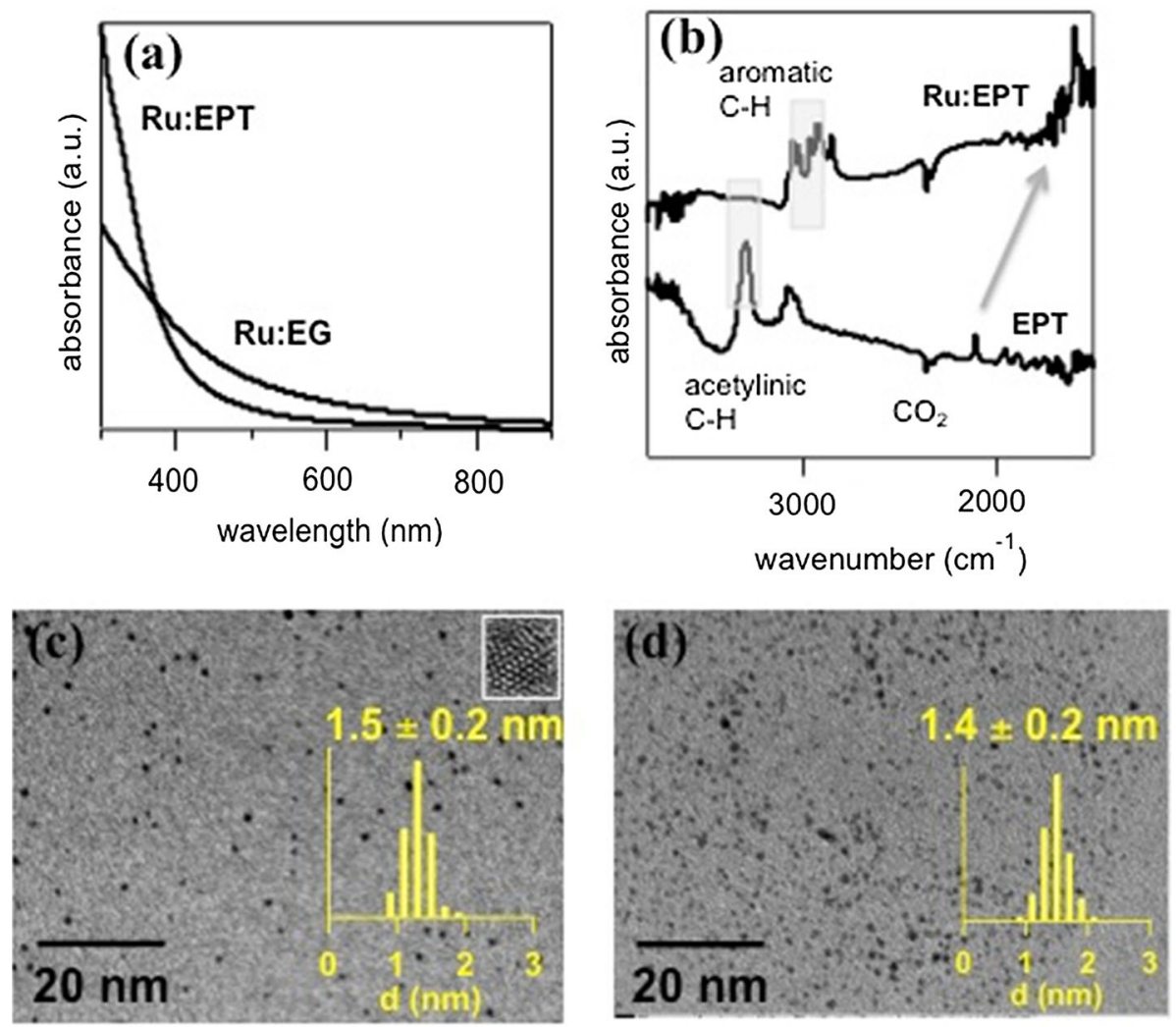

Figure 1. (a) UV-Vis spectra of Ru:EG and Ru:EPT; (b) FT-IR spectra of EPT and Ru:EPT; (c) TEM image and particle size distribution of Ru:EPT (inset shows HRTEM image of a single particle); (d) TEM image and Particle size distribution of Ru:EG.

$\mathrm{Ru} 3 \mathrm{p}_{1 / 2}$ and $3 \mathrm{p}_{3 / 2}$ orbital bonding energy at 460.5 and $483 \mathrm{eV}$ respectively (Figure 2c), which firmly proves the ' $\mathrm{Ru}$ ' atoms are essentially present at zero oxidation state. The powder-XRD profile of the Ru:EPT cluster was also measured to understand its crystalline nature and average crystallite size (Figure $2 \mathrm{~d}$ ). A very broad diffraction profile was observed, which reveals poor crystalline nature of the cluster material and its ultrasmall crystallite dimension. However, the peak position at $40^{\circ}$ ( $2 \theta$ value) indicates $\mathrm{Ru}$ (101) diffraction peak which coincides exactly with the bulk $\mathrm{Ru}$ diffraction profile. Since TEM image of the Ru:EPT cluster showed highly monodispersed particles, we were interested to get the molecular weight of the cluster through MALDI mass spectrometry using DCTB as a matrix. The systematic monitoring of MALDI mass spectra with increasing laser power (Figure $2 b$ ) showed a broad peak centered at 8600 Dalton. Thus, although the unanimous molecular composition the cluster could not be determined from this spectrum but we could confirm from TEM and MALDI analysis that the synthesized Ru-nanocluster is truly monodisperse with a molecular weight of around $8.6 \mathrm{kDa}$. Also in the precursor, Ru:EG cluster, the EG stabilizes Ru nanoparticles through weak chemisorption whereas EPT forms a strong metal carbon vinylidine bond in Ru:EPT, which results in highly stable and robust metal cluster.

\subsection{Optical and emission behavior of the cluster and metal-ligand binding motif}

The UV-Vis spectra of a water solution of Ru:EG cluster and DCM solution of Ru:EPT show similar pattern with an exponential rise towards lower wavelength (Figure 1a). This typical optical absorption pattern of the small metal particle is due to closely spaced Fermi level of nearly monodisperse clusters. The distinct UV-Vis spectra and emission spectra of EPT and Ru:EPT in DCM solution were measured as shown in Figure $3 \mathrm{a}$. The steady state solution phase fluorescence spectrum of EPT and Ru:EPT were measured by using $365 \mathrm{~nm}$ excitation wavelength light. Very interestingly, we could observe an $80 \mathrm{~nm}$ red shift of emission peak position of $\mathrm{Ru}: \mathrm{EPT}(457 \mathrm{~nm})$ as compared to free ligand $(377 \mathrm{~nm})$. In addition to that, the measured quantum yield value of the cluster $(\sim 7 \%)$ is 10 -fold higher than free ligand $(\sim 0.8 \%)$. This observation is very significant since luminescence of covalently attached ligand is drastically 

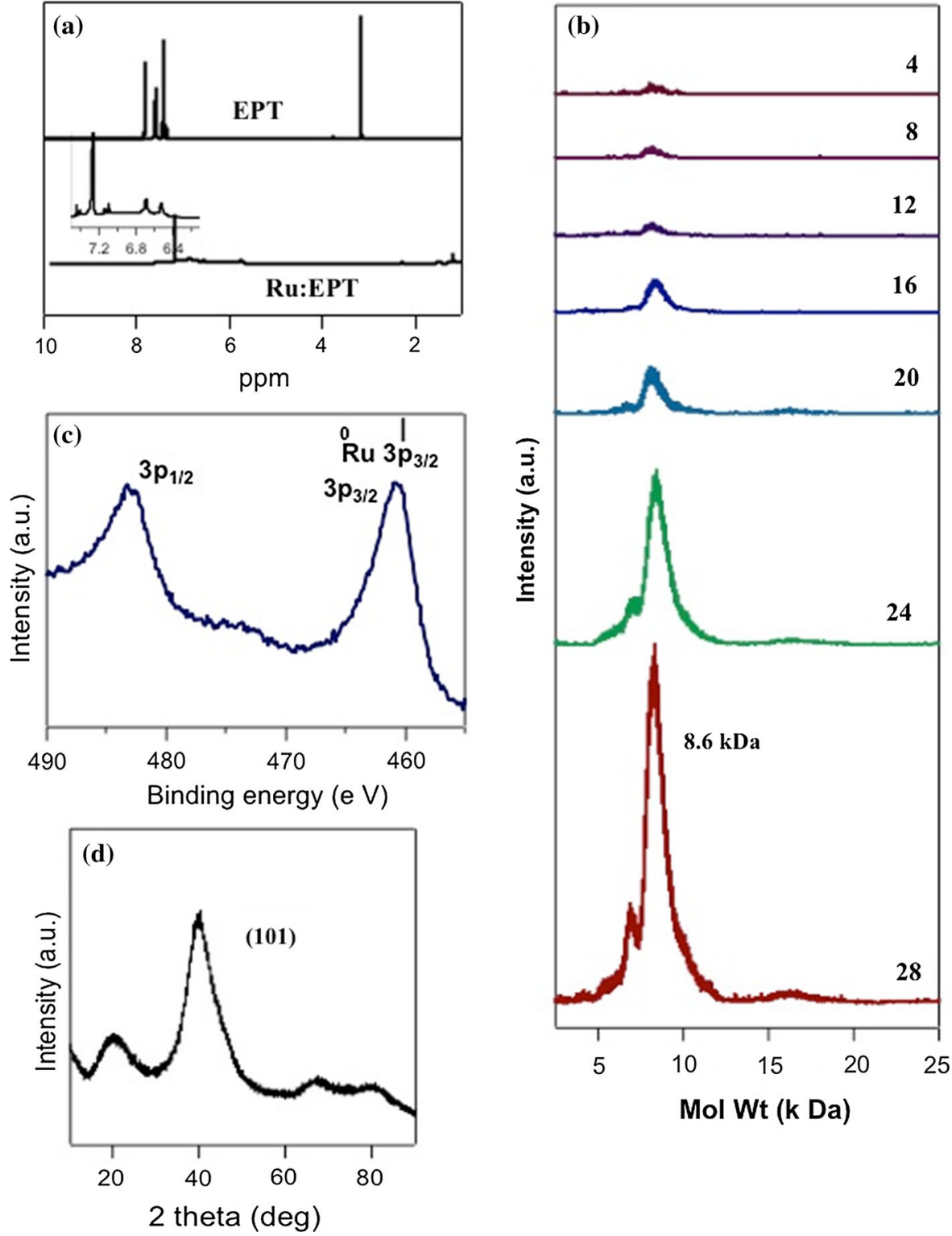

Mol Wt (k Da)

Figure 2. (a) ${ }^{1} \mathrm{H}$ NMR spectra of EPT and Ru:EPT; (b) Negative ion MALDI mass spectra of Ru:EPT with varying laser power (as indicated on the right side of each spectrum); (c) XPS spectra for Ru 3p orbital electron of Ru:EPT sample; (d) P-XRD profile of Ru:EPT.
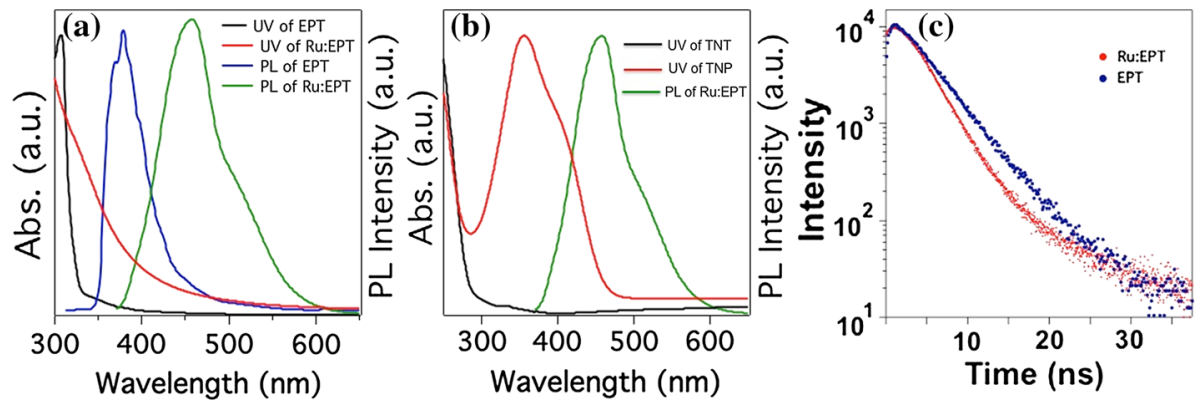

Figure 3. (a) Absorption and emission spectra of EPT and Ru:EPT as DCM solution at RT; (b) Absorption spectra of explosive (TNT and TNP) in DCM solution and emission spectra of Ru:EPT at RT; (c) PL decay profiles of EPT and Ru:EPT as DCM solution at RT. 

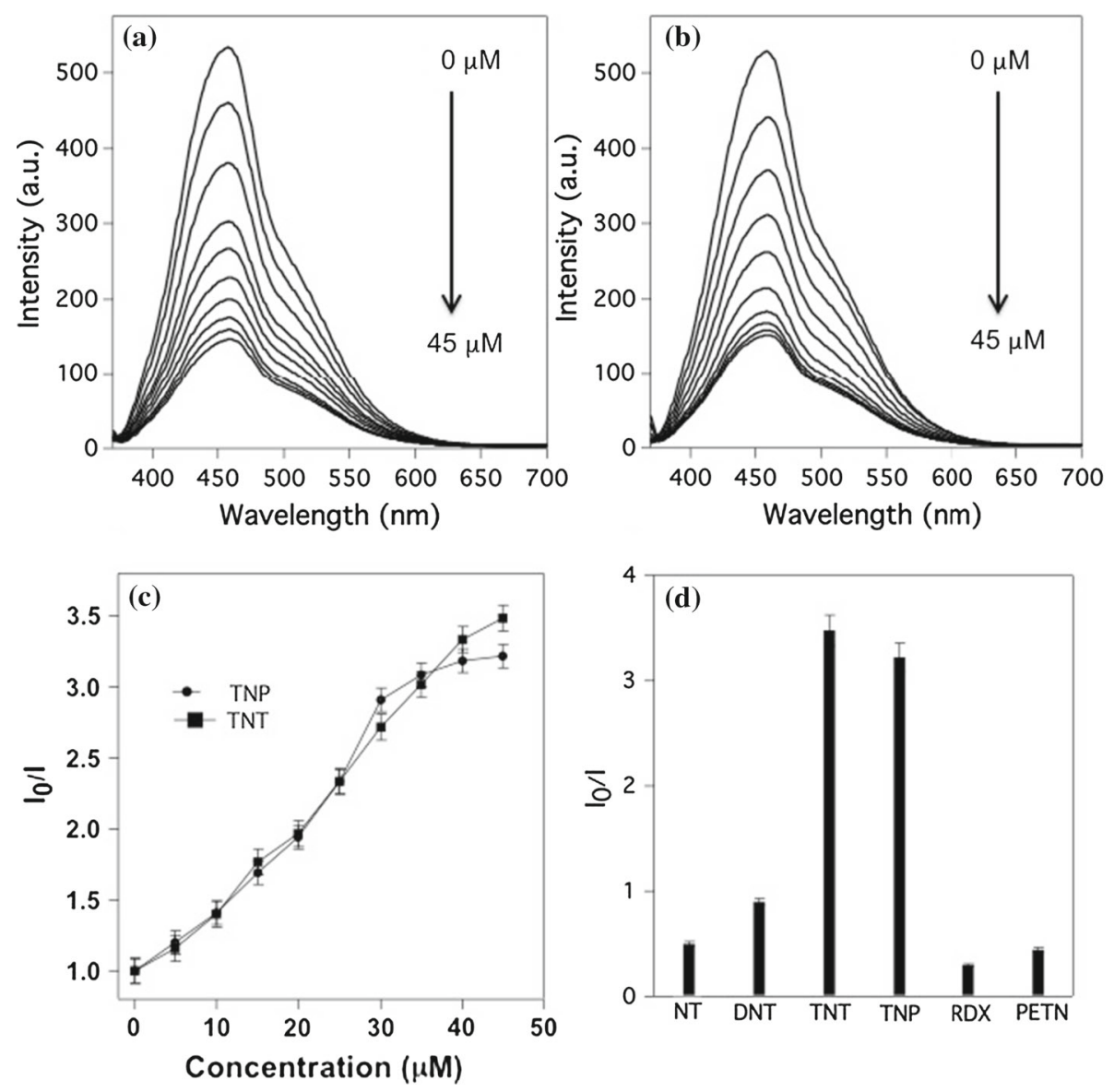

Figure 4. Fluorescence titration spectra of (a) TNT and (b) TNP with Ru:EPT in DCM; the concentrations of explosive molecules were varied from 0 to $45 \mu \mathrm{M}$ and a gradual decrease of peak intensity was observed. (c) Stern-Volmer plot obtained for the above titration results; (d) Bar diagram showing relative quenching efficiency of $\mathrm{Ru}: \mathrm{EPT}$ cluster for a variety of explosives in solution phase.

quenched by metal core through charge transfer from ligand to metal. ${ }^{16}$ The significant red-shifted emission behaviour and enhanced quantum yield of the cluster is due to the modified electronic structure of the ligand skeleton with strong perturbation from metalcore. This phenomenon indicates that the metal core acts as a conductor between phenanthrene moieties through $\mathrm{Ru}=\mathrm{C}=\mathrm{CHR}$ conjugated bonds and the aromatic ligands collectively behave as a conducting polymer. The emission lifetime measurement of the ligand and the cluster was also performed to understand the electronic energy level structures and are shown in Figure 3c. The emission lifetime was calculated to be 25 and 16 ns for EPT and Ru:EPT respectively and hence the lifetime of the ligand bonded to the cluster is substantially reduced as compared to free ligand since we believe the emission originates from ligand only. The facile charge transfer from EPT to Ru core through conjugated metal-carbon bond is thought to be responsible for the reduced lifetime of the excited state. ${ }^{32}$ The signature proton NMR signal, the redshifted FTIR stretching vibration of $-\mathrm{C} \equiv \mathrm{C}-$ bond, the presence of vibrational peaks characteristics of $\mathrm{C}\left(\mathrm{sp}^{2}\right)-\mathrm{H}$, the zero oxidation state of $\mathrm{Ru}$ through XPS characterization and the extreme redshifted luminescence feature indicates that $\mathrm{Ru}-\mathrm{C}$ binding motif of this cluster adopts vinylidene binding motif as opposed to alkynide motif of Au and Ir..$^{10,12}$

\subsection{Sensing study for nitroaromatic explosives}

By anticipating $\pi$ electron rich character of ligand skeleton, we were interested to investigate its effectiveness as fluorescence chemosensor for detection of nitroaromatic explosives. Different nitroaromatic explosive molecules (NT, DNT, TNT, TNP, RDX and PETN) were investigated for this purpose in solution (DCM) phase at RT. The steady state fluorescence titration measurements were carried out by mixing different concentration of these molecules to a DCM solution of Ru:EPT. As shown in Figures $4 a-b$, the fluorescence intensity of the cluster gradually decreases with the incremental addition of explosive molecules. The corresponding Stern-Volmer plots are also shown in Figure 4c with 
Stern-Volmer constant $\left(\mathrm{K}_{\mathrm{sv}}\right)$ values of $4.98 \times 10^{4} \mathrm{M}^{-1}$ and $4.79 \times 10^{4} \mathrm{M}^{-1}$ for TNT and TNP, respectively. Through systematic study with the incremental addition of analytes, we evaluated that this highly sensitive chemosensor could detect TNP and TNT efficiently with a concentration as low as $50 \mathrm{ppb}$. We ascribe that $\pi$ electron rich phenanthrene moiety interacts with $\pi$ electron deficient explosive molecules through $\pi-\pi$ stacking, which results in photoinduced electron transfer (PET) from excited donor state (phenanthrene) to acceptor (nitroaromatics) when irradiated with light. ${ }^{24}$ However, as shown in Figure 3b, the UV-Vis spectra of TNP and PL spectra of Ru:EPT overlap substantially, whereas the same does not happen with the optical spectrum of TNT. It has recently been demonstrated that FRETbased sensing mechanism is operative for nitroaromatic detection with proper selection of fluorophore. ${ }^{19} \mathrm{We}$ believe that for TNT and other nitroaromatic explosives, PET is the only responsible pathway for PL quenching but for TNP both PET and FRET are operative. However, as shown in Figure 4d, the quenching efficiency of the cluster is substantially diminished with other analytes (e.g., NT, DNT, RDX and PETN), which is due to their low pi-electron deficiency (NT, DNT) or having no pi-electron (RDX, PETN) character. These analytes could not form an effective dynamic adduct with photoexcited Ru:EPT skeleton through $\pi-\pi$ stacking and thus results in minimal PL quenching. A similar study was performed where only ligand (EPT) was used for sensing and showed much lower sensing performance $\left[\left(\mathrm{K}_{\mathrm{sv}}\right)\right.$ values of $\left.3.2 \times 10^{3} \mathrm{M}^{-1}\right]$ against titration with TNT in chloroform (Figure S3, Supplementary Information).

\section{Conclusions}

In summary, a new Ruthenium organometallic nanocluster with an average diameter of $1.5 \pm 0.2 \mathrm{~nm}$ and mol wt. of $\sim 8600$ Da was synthesized where a phenanthrene based alkyne ligand is bound to metal surface in a vinylidene motif. Contrary to extensive quenching of ligand emission by metalcore as usually observed, the ruthenium cluster showed intense luminescence, which originates from conjugated ligand structure. The metal core acts as a conductor to electronically conjugate the aromatic ligands and also its spherical curvature restricts lateral overlap of phenanthrene moiety to cause any excimer emission. This pi-electron rich phenanthrene ligand-stabilized $\mathrm{Ru}$ cluster showed high sensitivity for solution phase detection of nitroaromatic explosives through luminescence quenching method $\left(\mathrm{K}_{\mathrm{SV}}\right.$ up to $\left.4.98 \times 10^{4} \mathrm{M}^{-1}\right)$. A dynamic $\pi-\pi$ interaction between
Ru-bound phenanthrene moiety and nitroaromatic compounds followed by PET and or FRET is the possible mechanism behind this luminescence quenching.

\section{Supplementary Information (SI)}

Supplementary Information is available at www.ias.ac.in/ chemsci.

\section{Acknowledgements}

Financial assistance from Council of Scientific and Industrial Research (CSIR), New Delhi, India (Project no. 01(2873)/17/ EMR-II) is gratefully acknowledged.

\section{References}

1. Schmid G, Bäumle M, Geerkens M, Heim I, Osemann C and Sawitowski T 1999 Current and future applications of nanoclusters Chem. Soc. Rev. 28179

2. Shang L, Dong S and Nienhaus G U 2011 Ultra-small fluorescent metal nanoclusters: Synthesis and biological applications Nano Today 6401

3. Maity P, Xie S, Yamauchi M and Tsukuda T 2012 Stabilized gold clusters: From isolation toward controlled synthesis Nanoscale 44027

4. Jin R, Eahb S K and Peic Y 2012 Quantum-sized metal nanoclusters Nanoscale 44026

5. Mirkhalaf F, Paprotny J and Schiffrin D J 2006 Synthesis of metal nanoparticles stabilized by metal-carbon bonds J. Am. Chem. Soc. 1287400

6. Kang X, Zuckerman N B, Konopelski J P and Chen S 2010 Alkyne-stabilized ruthenium nanoparticles: Manipulation of intraparticle charge delocalization by nanoparticle charge states Angew. Chem. Int. Ed. 499496

7. Kang X, Zuckerman N B, Konopelski J P and Chen S 2012 Alkyne-functionalized ruthenium nanoparticles: Ruthenium-vinylidene bonds at the metal-ligand interface J. Am. Chem. Soc. 1341412

8. Maity P, Tsunoyama H, Yamauchi M, Xie S and Tsukuda T 2011 Organogold clusters protected by phenylacetylene J. Am. Chem. Soc. 13320123

9. Maity P, Wakabayashi T, Ichikuni N, Tsunoyama H, Xie S, Yamauchi M and Tsukuda T 2012 Selective synthesis of organogold magic clusters $\mathrm{Au}_{54}(\mathrm{C} \Xi \mathrm{CPh})_{26}$ Chem. Commun. 486085

10. Maity P, Takano S, Yamazoe S, Wakabayashi T and Tsukuda T 2013 Binding motif of terminal alkynes on gold clusters J. Am. Chem. Soc. 1359450

11. Srivastav A K, Agrawal B, Swami B, Agrawal Y K and Maity P 2107 Ligand exchange synthesis of organometallic $\mathrm{Rh}$ nanoparticles and application in explosive sensing J. Nanopart. Res. 19216

12. Yamamoto H, Maity P, Takahata R, Yamazoe S, Koyasu K, Kurashige W, Negishi Y and Tsukuda T 2017 Monodisperse iridium clusters protected by phenylacetylene: Implication for size-dependent evolution of binding sites J. Phys. Chem. C 12110936 
13. Wan X K, Tang Q, Yuan S F, Jiang D E and Wang Q M 2015 Au19 nanocluster featuring a V-shaped alkynylgold motif J. Am. Chem. Soc. 137652

14. Wang Y, Wan X K, Ren L, Su H, Li G, Malola S, Lin S, Tang Z, Häkkinen H, Teo B K, Wang Q M and Zheng N 2016 Atomically precise alkynyl-protected metal nanoclusters as a model catalyst: Observation of promoting effect of surface ligands on catalysis by metal nanoparticles J. Am. Chem. Soc. 1383278

15. Hong W, Li H, Liu S X, Fu Y, Li J, Kaliginedi V, Decurtins S and Wandlowski T 2012 Trimethylsilylterminated oligo(phenylene ethynylene)s: an approach to single-molecule junctions with covalent $\mathrm{Au}-\mathrm{C}$ o-bonds J. Am. Chem. Soc. 13419425

16. Thomas K G and Kamat P V 2003 Chromophorefunctionalized gold nanoparticles Acc. Chem. Res. 36 888

17. Yang J S and Swager T M 1998 Fluorescent porous polymer films as TNT chemosensors: Electronic and structural effects J. Am. Chem. Soc. 12011864

18. Sohn H, Sailor M J, Magde D and Trogler W C 2003 Detection of nitroaromatic explosives based on photoluminescent polymers containing metalloles J. Am. Chem. Soc. 1253821

19. Feng L, Li H, Qu Y and Lu C 2012 Detection of TNT based on conjugated polymer encapsulated in mesoporous silica nanoparticles through FRET Chem. Commun. 484633

20. Ma Y, Li H, Peng S and Wang L 2012 Highly selective and sensitive fluorescent paper sensor for nitroaromatic explosive detection Anal. Chem. 848415

21. Ma Y and Wang L 2014 Upconversion luminescence nanosensor for TNT selective and label-free quantification in the mixture of nitroaromatic explosives Talanta 120100

22. Ma Y, Huang S, Deng M and Wang L 2014 White upconversion luminescence nanocrystals for the simultaneous and selective detection of 2,4,6-trinitrotoluene and 2,4,6trinitrophenol ACS Appl. Mater. Interfaces 67790
23. Bai M, Huang S, Xu S, Hu G and Wang L 2015 Fluorescent nanosensors via photoinduced polymerization of hydrophobic inorganic quantum dots for the sensitive and selective detection of nitroaromatics Anal. Chem. 87 2383

24. Shanmugaraju S and Mukherjee P S $2015 \pi$-Electron rich small molecule sensors for the recognition of nitroaromatics Chem. Commun. 5116014

25. Wang S, Ma Y and Wang L 2015 Nanomaterials for luminescence detection of nitroaromatic explosives Trends Anal. Chem. 6513

26. Maity P, Bhatt A, Agrawal B and Jana A 2017 $\operatorname{pt}\left(\right.$ ii) $\mathrm{c}^{\wedge} \mathrm{n}^{\wedge} \mathrm{n}$-based luminophore/micelle adducts for sensing nitroaromatic explosives Langmuir 334291

27. Chen W, Zuckerman N B, Konopelski J P and Chen S 2010 Pyrene-functionalized ruthenium nanoparticles as effective chemosensors for nitroaromatic derivatives Anal. Chem. 82461

28. Zhang S, Han L, Li L, Cheng J, Yuan D and Luo J 2013 A highly symmetric metal-organic framework based on a propeller-like RU-organic metalloligand for photocatalysis and explosives detection Cryst. Growth Des. 135466

29. Crosby G A and Demas J N 1971 Measurement of photoluminescence quantum yields J. Phys. Chem. 75 991

30. Wang Y, Ren J, Deng K, Gui L and Tang Y 2000 Preparation of tractable platinum, rhodium, and ruthenium nanoclusters with small particle size in organic media Chem. Mater. 121622

31. Hostetler M J, Wingate J E, Zhong C J, Harris J E, Vachet R W, Clark M R, Londono J D, Green S J, Stokes J J, Wignall G D, Glish G L, Porter M D, Evans N D and Murray R W 1998 Alkanethiolate gold cluster molecules with core diameters from 1.5 to $5.2 \mathrm{~nm}$ : Core and monolayer properties as a function of core size Langmuir $\mathbf{1 4}$ 17

32. Berezin M Y and Achilefu S 2010 Fluorescence lifetime measurements and biological imaging Chem. Rev. 110 2641 